\title{
GROUPS WITH MANY PERMUTABLE SUBGROUPS
}

\author{
MARIO CURZIO, JOHN LENNOX, AKBAR RHEMTULLA and \\ JAMES WIEGOLD
}

(Received 6 December 1988; revised 1 August 1989)

Communicated by $\mathbf{H}$. Lausch

\begin{abstract}
We consider the influence on a group $G$ of the condition that every infinite set of cyclic subgroups of $G$ contains a pair that permute and prove (Theorem 1 ) that finitely generated soluble groups with this condition are centre-by-finite, and (Theorem 2) that torsion free groups satisfying the condition are abelian.
\end{abstract}

1980 Mathematics subject classification (Amer. Math. Soc.) (1985 Revision): 20 F 16.

\section{Introduction}

In response to a question of Paul Erdös, B. H. Neumann proved in [9] that a group is centre-by-finite if and only if the subsets consisting of mutually non-commuting elements are finite, and so these non-commuting sets are boundedly finite if they are finite. Extensions of problems of this type are to be found in [7] and [2].

We investigate here the following rather similar class of groups. Recall that a group is Hamiltonian if all of its subgroups are normal and it is quasiHamiltonian if every pair of subgroups permute (as sets). By obvious analogy, we say that a group $G$ is pseudo-Hamiltonian, or a PH-group, if the following conditions holds.

PH: Every infinite set of subgroups of $G$ contains a pair that permute.

We shall also be interested in the following related condition.

$\mathrm{PH}^{*}$ : Every infinite set of cyclic subgroups contains a pair that permute.

(C) 1990 Australian Mathematical Society 0263-6115/90 \$A2.00+0.00 
At first sight, this is a rather weaker condition than that mentioned above, and yet the group classes arising are not too different.

THEOREM 1. Every finitely generated soluble $\mathrm{PH}^{*}$-group is centre-by-finite.

THEOREM 2. All torsion-free $\mathrm{PH}^{*}$-groups are abelian.

Of course, all centre-by-finite groups satisfy $\mathrm{PH}$, though the converse is false. For example, Napolitani [5] and Iwasawa [8] have constructed quasiHamiltonian groups as follows:

$$
H_{n, q}=\left\langle a, b: a^{q^{n}}=1, b^{q^{n-1}}=1, a^{b}=a^{1+q}\right\rangle
$$

where $q$ is a prime and $n$ a positive integer. Clearly, the direct product of any number of groups of this type of coprime orders is quasi-Hamiltonian and thus satisfies $\mathrm{PH}$; and suitable constellations of the $n, q$ give rise to PH-groups that are not nilpotent and not centre-by-finite. Indeed, some infinite analogues of the Iwasawa-Napolitani groups are not even $F C$-groups (see [5] and [8]).

A number of problems about $\mathrm{PH}$-groups do not seem amenable to our methods. For instance, it should be the case that periodic PH-groups are locally finite, but the best we can say in this direction is the obvious fact that PH-groups of prime exponent are centre-by-finite, so that Tarski-Ol'shanskii monsters play no part in this subject. This is because the permutable product of two groups of the same prime order is commutative, so that the results of [9] apply. On the other hand, there are infinite groups of exponent $p^{2}$ with just $(p-1)$ elements of order $p$; for example, a suitable factor-group of Adian's group [1, page 269] is of this type, and is certainly not a PH-group.

We thank the referee for some useful comments.

\section{Proofs}

We break down the proof of Theorem 1 into several steps.

2.1. Let $G$ be a finitely generated soluble group such that for every cyclic subgroup $H$ and every element $x$ of $G$, there exists a positive integer $i$ such that

$$
H H^{x^{i}}=H^{x^{i}} H \text {. }
$$

Then $G$ is nilpotent-by-finite.

Proof. For arbitrary $x, y$ in $G$, there must exist $i>0$ such that

$$
\langle y\rangle\langle y\rangle^{x^{i}}=\langle y\rangle^{x^{i}}\langle y\rangle \text {. }
$$


Thus, by [3], the product $K=\langle y\rangle\langle y\rangle^{x^{i}}$ has a torsion-free abelian subgroup of finite index. Thus $\left[y^{m},\left(y^{m}\right)^{x^{i}}\right]=1$. for some $m \geq 1$, so

$$
\left[x^{i}, y^{m}, y^{m}\right]=1
$$

for some $m \geq 1$ and some $i>0$.

To complete the proof of 2.1 , we need the following vital result.

2.2. Let $G$ be a finitely generated soluble group such that for all $x, y$ in $G$ there exist integers $n, i \geq 1$, such that $\left[x^{i}, y^{n}, y^{n}\right]=1$. Then $G$ is nilpotent-by-finite.

Proof. By induction on the solubility length of $G$, we may assume that $G$ is abelian-by-nilpotent-by-finite, and thus, ignoring the finite factor at the top, that $G$ is abelian-by-nilpotent. Thus $G$ is eremitic [6]; this means that there is an integer $e \geq 1$ depending only on $G$ such that $\left[u, v^{e}\right]=1$ whenever $\left[u, v^{m}\right]=1$ for elements $u, v$ of $G$ and an integer $m>1$. Let $A$ be an abelian normal subgroup of $G$ such that $G / A$ is nilpotent. For $a \in A$ and $y \in G$, we have $\left[(y a)^{i}, y^{n}, y^{n}\right]=1$ for some $i, n>1$, so, after a small calculation, we have $\left[b, y^{n}, y^{n}\right]=1$, where $b=a^{1+y+y^{2}+\cdots+y^{i-1}}$. But $\langle a, y\rangle$ is metabelian, so $\left[b, y^{e}, y^{n}\right]=1$ and $\left[b, y^{e}, y^{e}\right]=1$. Write $A$ additively; then $a\left(1+y+\cdots+y^{i-1}\right)\left(1-y^{e}\right)^{2}=0$, so that multiplying by $1-y$ gives $a\left(1-y^{i}\right)\left(1-y^{e}\right)^{2}=0$, and so by eremiticity, $a\left(1-y^{e}\right)^{3}=0$.

It will be enough if we show that $G^{e}$ is nilpotent, since it is of finite index. All the elements of the form $y^{e}$ act nilpotently on $A \cap G^{e}$, so $\left\langle A \cap G^{e}, y^{e}\right\rangle$ is nilpotent. But this subgroup is subnormal in $G^{e}$, so $G^{e}$ is locally nilpotent and thus nilpotent since it is finitely generated.

Note that 2.2 remains true if the hypothesis is changed to $\left[x^{i}, y^{n}, \ldots, y^{n}\right]$ $=1$, where the repeated commutator has length depending on $x$ and $y$ only. We thus have a generalisation of the well-known fact that a finitely generated soluble Engel group is nilpotent.

Our final preliminary is

\subsection{Every torsion-free nilpotent $\mathrm{PH}^{*}$-group is abelian.}

Proof. We may assume that $G$ is 2-generator, say $G=\langle x, y\rangle$. Let $Z$ be the centre of $G$. Then $G / Z$ is torsion-free and so by an obvious induction, it is abelian and hence $G$ is of class 2 . Setting $H_{i}=\left\langle x y^{i}\right\rangle, i \in \mathbb{Z}$, we have $H_{n} H_{m}=H_{m} H_{n}$ for some $n, m>0, n \neq m$ so $H_{n} H_{m}$ is a metabelian group by Itô's Theorem and by [3] has an abelian subgroup of finite index. But this group is also torsion-free nilpotent of class 2 and an easy argument 
shows that it is abelian. Hence $x y^{n}$ and $x y^{m}$ commute, and once again, the fact that $G$ is torsion-free nilpotent of class 2 gives that $x$ and $y$ must commute.

We are now in a position to prove Theorem 1 . Let $G$ be a finitely generated soluble $\mathrm{PH}^{*}$-group. By $2.1, G$ is nilpotent-by-finite, so it has a torsion-free nilpotent subgroup $A$ of finite index; by $2.3, A$ is abelian. We proceed by induction on $|G / A|$, all being well when $G=A$.

If $\langle A, x\rangle\langle G$ for all $x \in G$, then $\langle A, x\rangle$ is an $F C$-group for all $x$ in $G$, so $G$ is an $F C$-group. Finitely generated $F C$-groups are centre-by-finite and thus we may assume that $\langle A, x\rangle=G$ for some $x$. Let $n$ be the order of $x$ modulo $A$. If $n$ is not a prime power, then $n=r s$ with $(r, s)=1$. Since $\left\langle A, x^{r}\right\rangle$ and $\left\langle A, x^{s}\right\rangle$ are proper subgroups of $G$, the centralizers $C_{G}\left(x^{r}\right)$ and $C_{G}\left(x^{s}\right)$ are of finite index in $G$, as above, and $C_{G}(x)>C_{G}\left(x^{r}\right) \cap C_{G}\left(x^{s}\right)$.

We know now that $n=p^{m}$ for some prime $p$. By induction, $\left\langle A, x^{p}\right\rangle$ is centre-by-finite. Thus $\left[A, x^{p}\right]$ is finite; since $A$ is torsion-free and normal, this means that $\left[A, x^{p}\right]=1$. Thus the group $B:=\left\langle A, x^{p}\right\rangle$ is abelian, and of course $x^{p}$ is in the centre of $G$.

We can assume that $x$ has infinitely many conjugates in $G$, else the centre $Z(G)$ has finite index, since it contains $A \cap C_{G}(x)$. Thus, there must exist an $a$ such that $\left\langle x^{a^{i}}\right\rangle \neq\left\langle x^{a^{j}}\right\rangle$ if $i \neq j$. Property PH $^{*}$ now means that $y:=\langle x\rangle\left\langle x^{b}\right\rangle=\left\langle x^{b}\right\rangle\langle x\rangle$ for some $b=a^{i}$. Modulo the central subgroup $\left\langle x^{p}\right\rangle, Y$ has order $p$ or $p^{2}$, so $[x, b]^{p} \in\left\langle x^{p}\right\rangle$; since $[x, b]^{p}=\left[x, b^{p}\right]$, we have the contradiction that $\left\langle x^{b^{p}}\right\rangle=\langle x\rangle$. Thus $x$ has only finitely many conjugates after all, and $G$ is centre-by-finite. This completes the proof of Theorem 1.

Proof of Theorem 2. We are required to show that a torsion-free $\mathbf{P H}^{*}$ group $G$ is abelian. For this purpose it is sufficient to assume that $G=$ $\left\langle g_{1}, \ldots, g_{k}\right\rangle$ is finitely generated. Suppose that $a, b$ are elements of $G$ such that $\langle a\rangle\langle b\rangle=\langle b\rangle\langle a\rangle$. Since this product is metabelian, it follows from Theorem 1 and the fact that $G$ is torsion free that $\langle a, b\rangle$ is abelian. Now for any pair $x, y$ of elements of $G$, there exists $i>0$ such that $\langle y\rangle\left\langle y^{x^{i}}\right\rangle=\left\langle y^{x^{i}}\right\rangle\langle y\rangle$. Thus $\left\langle y, y^{x^{i}}\right\rangle$ is abelian. Similarly, $\left\langle x, x^{y^{j}}\right\rangle$ is abelian for some $j>0$. Hence $\left\langle x^{i}, y^{j}\right\rangle$ is nilpotent and hence abelian by Theorem 1. Since $\left[x^{i}, y, y\right]=1$, we have $1=\left[x^{i}, y^{j}\right]=\left[x^{i}, y\right]^{j}$, so $\left[x^{i}, y\right]=1$. In particular, by considering the pairs $\left(x, g_{j}\right)$, for $j=1, \ldots, k$, we get $\left[x^{t}, G\right]=1$ for some $t>0$. This shows that $G / Z(G)$ is periodic.

Obtain, if possible, a sequence $\left(a_{1}, a_{2}, \ldots\right)$ of elements of $G$ as follows. Pick any $a_{1} \in G \backslash Z(G)$ and for $i \geq 2$, pick $a_{i}$ from $G \backslash \bigcup_{j=1}^{i-1} C_{G}\left(a_{j}\right)$. If $\bigcup_{j=1}^{n} C_{G}\left(a_{j}\right)=G$ for some $n \in N$, then $C_{G}\left(a_{i}\right)$ is of finite index in $G$ for 
some $i \leq n$. Set $A=\left\langle a_{i}^{G}\right\rangle$. Then $A$ is in the $F C$-centre of $G$ and $[A, G]$ is finite. But $G$ is torsion-free, whence $A \leq Z(G)$, contradicting our choice of $a_{i}$. We conclude that in this case $G$ is abelian.

The other alternative is the existence of an infinite sequence $\left(a_{1}, a_{2}, \ldots\right)$ as constructed above. By hypothesis, $\left\langle a_{j}\right\rangle\left\langle a_{i}\right\rangle=\left\langle a_{i}\right\rangle\left\langle a_{j}\right\rangle$ for some $0<i<j$. In this case $\left\langle a_{i}, a_{j}\right\rangle$ is abelian, as shown earlier in the proof. But then $a_{j} \in C_{G}\left(a_{i}\right)$, a contradiction. This completes the proof of Theorem 2 .

\section{References}

[1] S. Adian, The Burnside problem and identities in groups, (Ergeb. Math. Grenzgeb., Springer-Verlag, Berlin, Heidelberg and New York, 1979).

[2] J. R. J. Groves, 'A conjecture of Lennox and Wiegold concerning supersoluble groups', J. Austral. Math. Soc. 35 (1983), 218-220.

[3] Hermann Heineken and John C. Lennox, 'A note on products of abelian groups', Arch. Math. 41 (1983), 498-501.

[4] N. Îto, 'Über das Produkt von zwei abelschen Gruppen', Math. Z. 62 (1955), 400-401.

[5] K. Iwasawa, Über die endlichen Gruppen und die Verbände ihrer Untergruppen, J. Fac. Sci. Univ. Tokyo Sect. IA Math. 4 (1941), 171-199.

[6] J. C. Lennox and J. E. Roseblade, 'Centrality in finitely generated soluble groups', $J$. Algebra 16 (1970), 399-435.

[7] J. C. Lennox and James Wiegold, 'Extensions of a problem of Paul Erdos on groups', $J$. Austral. Math. Soc. 31 (1981), 459-463.

[8] F. Napolitani, 'Sui p-gruppi modulari finiti', Rend. Sem. Mat. Univ. Padova 39 (1967), 296-303.

[9] B. H. Neumann, 'A problem of Paul Erdös on groups', J. Austral. Math. Soc. 21 (1976), 467-472.

Dipartimento di Matematica

Pura e Applicata

Università Degli Studi di Napoli

Napoli

Italia

Department of Mathematics

University of Alberta

Edmonton, Alberta

Canada
School of Mathematics

University of Wales

College of Cardiff

Cardiff CF2 4AG

Wales

School of Mathematics

University of Wales

College of Cardiff

Cardiff CF2 4AG

Wales 